\title{
Optimization of commercial net spacers in spiral wound membrane modules
}

\author{
F. Li*, W. Meindersma, A.B. de Haan, T. Reith \\ Department of Chemical Engineering, University of Twente, P.O. Box 217, 7500 AE Enschede, The Netherlands
}

Received 4 February 2002; received in revised form 22 May 2002; accepted 31 May 2002

\begin{abstract}
CFD simulations have been used to determine mass transfer coefficients and power consumption of commercial net spacers. The simulations show transversal and longitudinal vortices, vortex shedding and instationary flow behavior leading to the enhanced mass transfer in spacer filled-channels compared to empty channels. The results of the simulations were validated with experiments and compared with data reported in literature, showing satisfactory agreement. Furthermore, CFD simulations were used to optimize the geometry of commercial net spacers in terms of mass transfer and power consumption. The performance of these optimized spacer geometries will be used as reference for future work on the development of new high-performance spacer shapes.
\end{abstract}

() 2002 Elsevier Science B.V. All rights reserved.

Keywords: Spiral wound module; Membrane filtration; Spacer; Optimization; CFD

\section{Introduction}

In membrane processes accumulation of rejected species near the feed side of the membrane (concentration polarization) causes reduction of permeate flux and deterioration of permeate quality. Accumulation of rejected species can be suppressed by creating back-mixing from the membrane to the bulk of the liquid. Various hydrodynamic approaches have been developed for that purpose.

Gupta et al. [1] showed that helically baffled cross-flow is very effective in tubular membrane modules, and that more than $50 \%$ enhancement in permeate flux can be obtained at the same power dissipation compared to modules without baffles. It

\footnotetext{
* Corresponding author. Tel.: +31-53-489-5447; fax: +31-53-489-4821.

E-mail address: f.li@ct.utwente.nl (F. Li).
}

was found experimentally by Yeh and Chen [2] that high permeation fluxes could be obtained in tubular membrane modules by inserting round rods wrapped with wires. Without major increase in power dissipation, Millward et al. [3] found that significant mass transfer enhancement could be achieved under laminar flow conditions inside a vortex-wave membrane bio-reactor.

Van der Waal and Racz [4] have demonstrated experimentally that corrugations on the membrane surface improve back-mixing, provided that they are not too close together. Similar results have been reported for electrochemical cells with obstacles creating mixing [5-8].

Most of the hydrodynamic approaches share the same mechanism: obstacles near or on the membrane surface create back-mixing from the membrane surface to the bulk of the liquid feed flow. These obstacles are often referred to as "turbulence promoters" 


\begin{tabular}{|ll}
\hline Nomenclature \\
$A$ & area of membranes $\left(\mathrm{m}^{2}\right)$ \\
$C_{\mathrm{b}}$ & bulk concentration $\left(\mathrm{kg} / \mathrm{m}^{3}\right)$ \\
$C_{\mathrm{w}}$ & concentration at membrane \\
& surfaces $\left(\mathrm{kg} / \mathrm{m}^{3}\right)$ \\
$\partial C / \partial y$ & concentration gradient at membrane \\
& surfaces $\left(\mathrm{kg} / \mathrm{m}^{4}\right)$ \\
$d$ & diameter of filaments $(\mathrm{m})$ \\
$\mathcal{D}$ & diffusivity $\left(\mathrm{m}^{2} \mathrm{~s}^{-1}\right)$ \\
$E u$ & Euler number \\
$f_{\text {mod }}$ & modified friction factor \\
$h$ & channel height $(\mathrm{m})$ \\
$k$ & local mass transfer coefficient $(\mathrm{m} / \mathrm{s})$ \\
$k_{\mathrm{m}}$ & average mass transfer coefficient $(\mathrm{m} / \mathrm{s})$ \\
$L$ & channel length $(\mathrm{m})$ \\
$l$ & filament spacing $(\mathrm{m})$ \\
$l_{1}$ & filament spacing $(\mathrm{m})$ \\
$l_{2}$ & filament spacing $(\mathrm{m})$ \\
$P n$ & power number \\
$R e$ & Reynolds number \\
$S c$ & Schmidt number \\
$S h$ & Sherwood number \\
$\mathrm{SPC}$ & specific power consumption $(\mathrm{Pa} / \mathrm{s})$ \\
$u$ & mean velocity $(\mathrm{m} / \mathrm{s})$ \\
$x$ & $x$-coordinate \\
$y$ & $y$-coordinate \\
$z$ & $z$-coordinate \\
$G r e e k$ & letters \\
$\alpha$ & flow attack angle \\
$\beta$ & angle between crossing filaments \\
$\mu$ & dynamic viscosity $(\mathrm{Pas})$ \\
$\rho$ & density $\left(\mathrm{kg} / \mathrm{m}^{3}\right)$ \\
$v$ & kinematic viscosity $\left(\mathrm{m}^{2} / \mathrm{s}\right)$ \\
$\Delta p / L$ & pressure gradient in mean flow \\
& direction $(\mathrm{Pa} / \mathrm{m})$ \\
& \\
&
\end{tabular}

but "vortex promoters" would be more appropriate, since flows inside membrane modules are often characterized with vortex shedding.

Many studies on heat transfer enhancement have been reported, which are of interest due to the similarity between heat and mass transfer. A technology review by Wang [9] indicates that louver and slit shaped surfaces are effective means for enhancing heat transfer in fin-and-tube heat exchangers. Fiebig [10] studied various types of vortex generators (promoters) in a flat channel and concluded that generators of longitudinal vortices were very effective for heat transfer enhancement.

In spiral wound modules, spacers are used to provide mechanical support for the membranes, but they also act as vortex promoters in the feed channels. Experimental studies [11-15] have shown that spacers enhance mass transport (back-mixing) in feed channels of spiral wound modules, but that spacers also increase flow resistance. The work of Schock and Miquel [12] should be mentioned here, since they had measured mass transfer and flow resistance in flat channels and spiral wound modules both filled with various commercial spacers, some of which were similar to the spacers investigated in the present study. Their results are compared with our results in Fig. 11. They noted that spacer-filled flat channels and channels in spiral wound modules with the same spacers exhibited similar flow characteristics. This means that pressure drops and mass transfer coefficients measured in flat channels are also valid for spiral wound modules.

CFD simulation is a powerful tool for the study of flow patterns in spacer-filled channels. A number of interesting studies have been published since the late 1990s. These CFD simulations confirm that the geometric parameters of spacers are major variables having great influence on flow resistance and mass transfer [16,17].

Reports describing combined modeling and experimental investigations into the performance and optimization of net spacers were not found in the literature.

Presently net spacers are commonly used in the feed channels of commercial spiral wound modules. Two types of commercial net spacers are frequently used: woven and non-woven spacers as shown in Fig. 1.

It is clear that back-mixing is more effective in spacer-filled channels than in empty channels, but that also the pressure drop over the feed channel, i.e. the mechanical energy dissipation in the feed channel is higher in spacer-filled channels. Therefore, it is necessary to optimize the spacer shape in order to obtain an optimal performance in terms of mass transfer and mechanical energy dissipation. An analysis by Costa 

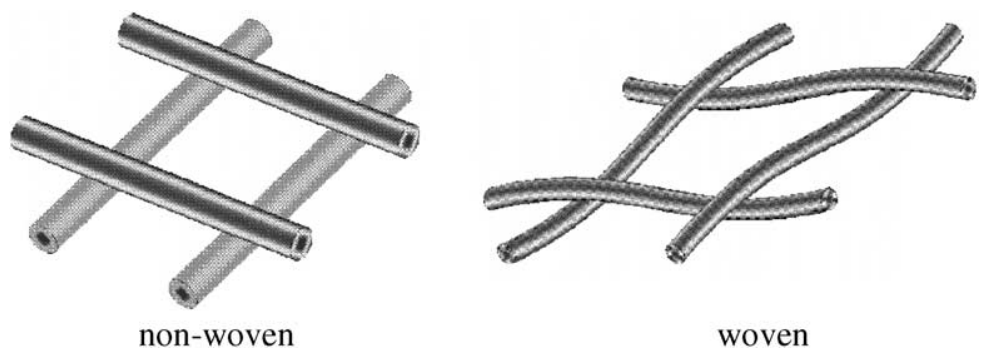

Fig. 1. The basic shapes of commercial net spacers.

et al. [18] of the processing costs in spacer-filled channels, including equipment and energy consumption, demonstrates that there are distinct possibilities to optimize spacer geometries.

It is obvious from the above introduction that there is ample room for a systematic experimental and modeling investigation of spacer optimization in terms of mass transfer and energy dissipation.

Spacer geometry optimization by CFD simulation and experimental determination of mass transfer in ducts is the subject of the research project "Spacer Optimization" started in 1999 at the Separation Technology Group of the University of Twente. The present publication focuses on one particular aspect, i.e. the optimization of spacer geometry of commercial non-woven net spacers by CFD simulation. The performance of optimized commercial net spacers will serve as the standard of comparison against which newly developed spacer geometries will be judged.

The present paper focuses on calculations of power dissipation and mass transfer in flat channels filled with net spacers using CFD simulation methods. Since the simple geometry of non-woven net spacers facilitate CFD simulation, woven net spacers are not studied. There is no loss of generality, since both spacer types give similar results in terms of mass transfer and power dissipation.

Commercial spiral wound membrane modules are normally operated at low-Reynolds numbers $(R e=$ $100-500)$. It was decided to optimize spacer performance in the same range of low-Reynolds numbers, since it may be expected that optimization is most effective in low-Reynolds flows. The Schmidt number in the calculations was the same as the Schmidt number used in our experimental studies $(S c=1278)$.

\section{Geometric parameters of non-woven net spacers}

A non-woven spacer as shown in Fig. 1, can be characterized by four parameters as shown in Fig. 2: the distance between spacer filaments $l_{1}$ and $l_{2}$, the angle between the spacer filaments $\beta$ and the flow attack angle $\alpha$. In this study, we confine ourselves to cylindrical filaments. The diameter of the filaments $(d)$ is half of the channel's height. For commercial spacers, $l_{1}$ is about equal to $l_{2}$, i.e. $l_{1}=l_{2}=l$.

The influence of the four geometric parameters on mass transfer enhancement and mechanical energy dissipation are investigated in the following parameter ranges:

(1) Angle $\alpha$ and $\beta$ are kept $90^{\circ}$, and the values of 2, $4,6,8$ and 10 will be assigned to $l / h$.

(2) The influence of the flow attack angle $\alpha$ is studied with values of $0,15,30$ and $45^{\circ}$ for the optimal ratio of $l / h$ found in the first step and $\beta=90^{\circ}$.

(3) With the optimal ratio of $l / h$ found in the first step, the following combination of $\alpha$ and $\beta$ will be used to study the influence of $\beta$ :

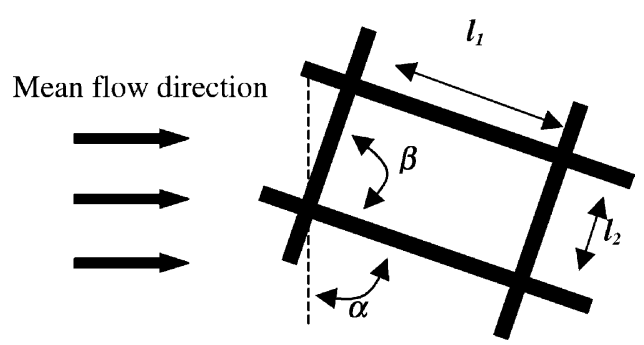

Fig. 2. The geometric characterization of a non-woven spacer. 
(i) $\beta=60^{\circ}, \alpha=60^{\circ}$; and

(ii) $\beta=120^{\circ}$ and $\alpha=30^{\circ}$.

\section{Optimization and scale-up}

\subsection{Optimization of spacers}

It is obvious that the optimization of spacer performance should be based on evaluation of economic parameters like investment cost of the installation, cross-flow pumping energy, maintenance cost, personnel cost, etc. Since an economic evaluation is outside the scope of the present study, a simple comparison method of spacer performance will be used.

Spacers can result in enhanced mass transfer from the membrane wall to the liquid bulk (back-mixing). Therefore, in the present study, spacer performance will be expressed in terms of mass transfer coefficients, $k_{\mathrm{m}}$. Since cross-flow pumping power consumption is a major cost item, the performance of a spacer in terms of $k_{\mathrm{m}}$ will be compared on the basis of mechanical power consumption dissipated per $\mathrm{m}^{3}$ channel specific power consumption (SPC):

$\mathrm{SPC}=\frac{\Delta P u A}{L A}=\frac{\Delta P}{L} u$

where $\Delta P$ is the pressure drop over the channel, $L$ is the channel length, $A$ is the cross-section area of the channel, and $u$ is the mean liquid velocity in the channel.

A similar approach was described by Storck and Hutin [19] in which results were presented in the plots of $k_{\mathrm{m}}$ versus SPC.

Although the latter representation is suitable for comparison purposes, it is less suited for scale-up (or scale-down) from experimental to industrial dimensions and flow conditions. For that purpose a dimensional analysis of the process is needed. For details on dimensional analysis the reader is referred to Zlokarnik [20].

\subsection{Dimensional analysis}

The mass transfer coefficient, $k_{\mathrm{m}}$, taken as an average over the channel length, $L$, is dependent on density, $\rho$, dynamic viscosity, $\mu$ and diffusivity, $\mathcal{D}$, of the liquid, height of the channel, $h$, the distance between the spacer filaments, $l$, the flow attack angle, $\alpha$, the angle between the spacer filaments, $\beta$, and liquid velocity $u$ :

$k_{\mathrm{m}}=F(\mathcal{D}, \rho, \mu, h, l, \alpha, \beta, u)$

The definition of the mass transfer coefficient, $k_{\mathrm{m}}$, is discussed in Section 4.

Dimensional analysis leads to the following relationship between dimensionless numbers:

$S h=F\left(\operatorname{Re}, S c, \frac{l}{h}, \alpha, \beta\right)$

in which

$S h \equiv \frac{k_{\mathrm{m}} h}{\mathcal{D}}$

$R e \equiv \frac{\rho u h}{\mu}$

$S c \equiv \frac{\mu}{\rho \mathcal{D}}$

The SPC is described by the physical relationship

$\mathrm{SPC}=F(\rho, \mu, h, l, \alpha, \beta, u)$

Eq. (7) becomes after dimensional analysis:

$P n=F\left(\operatorname{Re}, \frac{l}{h}, \alpha, \beta\right)$

in which $P n$ is the dimensionless power number:

$P n \equiv \operatorname{SPC} \frac{\rho^{2} h^{4}}{\mu^{3}}$

In Section 4, it is shown that the non-woven net spacers have streamwise-periodic cross-sections, resulting in periodic fully-developed flows along the greater part of the channel. This means that the mass transfer coefficient, $k_{\mathrm{m}}$, is independent of the channel length, $L$. Therefore, $L$ is not a relevant variable in the dimensional analysis and appears only in combination with $\Delta P$ as $\Delta P / L$.

It should be realized that:

$P n=\operatorname{SPC} \frac{\rho^{2} h^{4}}{\mu^{3}}=\frac{\Delta P}{\rho u^{2}} \frac{h}{L}\left(\frac{\rho u h}{\mu}\right)^{3}=E u \frac{h}{L} R e^{3}$

with

$E u \equiv \frac{\Delta P}{\rho u^{2}}$ 
Eq. (8), therefore, reduces to the well-known expression (12):

$E u \frac{h}{L}=F\left(\operatorname{Re}, \frac{l}{h}, \alpha, \beta\right)$

where $E u(h / L)$ can be recognized as the Fanning friction factor, $f$.

Elimination of $R e$ from Eqs. (3) and (8) results in the dimensionless relationship:

$S h=F\left(P n, S c, \frac{l}{h}, \alpha, \beta\right)$

Storck and Hutin [19] obtained a similar relationship as Eq. (13). Issacson and Sonin [21] have compared the performance of spacers on the basis of a modified friction factor, $f_{\text {mod }}$ :

$f_{\text {mod }} \equiv \operatorname{Re}\left(E u \frac{h}{L}\right)^{1 / 3}=P n^{1 / 3}$

It is obvious that this modified friction factor does not give new insights in the physical background of the process and is, therefore, not used in the present study.

Eqs. (3), (8), (12) and (13) relate dimensionless numbers and can consequently be used for purposes of scale-up (or scale-down) to industrial conditions. An additional clear advantage of the application of dimensional analysis is that with a limited number of simulations a wide range of geometrical and operational variables and physical properties can be covered.

In the present study, the average mass transfer coefficients, $k_{\mathrm{m}}$, will be presented in $S h$ versus $P n$ plots with $l / h, \alpha$ and $\beta$ as parameters, in accordance with Eq. (13).

\section{Modeling and numerical methods}

Channels with non-woven spacers have streamwiseperiodic cross-sections. This means that the channel consists of a large number of identical cells. A cell for the non-woven net spacer is as shown in Fig. 3. In a recent paper of Yuan et al. [24], it was shown that the flow and heat transfer in channels with streamwise-periodic cross-sections became 'periodic fully-developed' after a few cycles or cells. This finding applies also to the mass transfer in channels with non-woven net spacers, as was confirmed during our experimental studies (to be published in a future paper). This means that after a few cells local mass transfer coefficients in a spacer-filled channel become fully periodic and that the mass transfer coefficients averaged over cell area are equal in each cell. Consequently, average mass transfer coefficients are not dependent on channel length, $L$. This means that the simulation of flow and mass transfer viz. the computational domain can be limited to one cell to which periodic boundary conditions are applied. A computational domain, which corresponds to a spacer with $l / h=4, \alpha=90^{\circ}$ and $\beta=90^{\circ}$, is as shown in Fig. 3.

The following boundary conditions are applied to the computational domain:

- On the filament surfaces liquid velocities are zero.

- On the two membrane surfaces (top and bottom walls of the channel) liquid velocities and concentrations are zero.

- In the four remaining vertical cell boundaries two pairs of opposite flow cross-sections can be distinguished. For each pair periodic boundary conditions are specified.

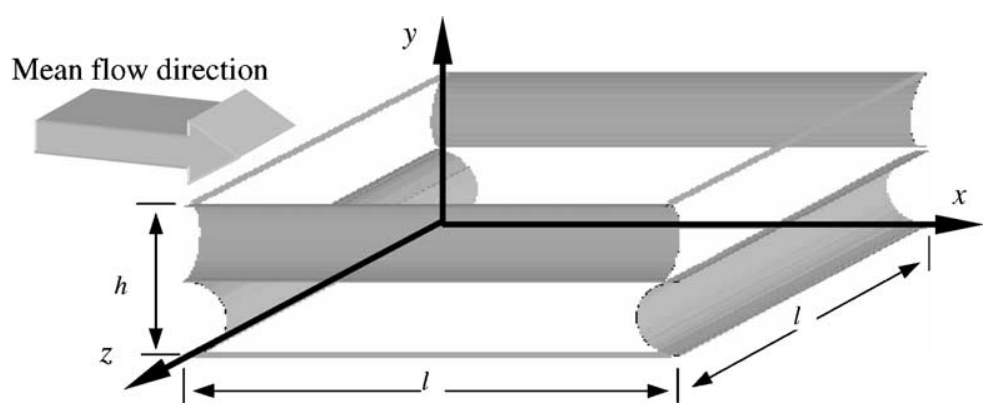

Fig. 3. Computational domain with $l / h=4, \alpha=90^{\circ}, \beta=90^{\circ}$. 
The flow in a spacer-filled flat channel is 3D and mass transfer takes place on the walls of the channel. The Navier-Stokes flow equations and the mass transport equation each with its boundary conditions are solved simultaneously with the CFX-4.3 software package, a CFD simulator from AEA technology. In the present study, the Navier-Stokes flow equations and the mass transport equation were solved directly, without using turbulence models. The following methods have been used for the simulations:

- The backward Euler differencing scheme, which is fully implicit in time, is used for solving the time dependent differential equations;

- The diffusive terms in all equations are discretized with the central differencing scheme (CDS);

- The convective terms are discretized with the higher-order upwind differencing scheme (HUD);

- A fixed time-step $\Delta t=0.025 \mathrm{~s}$ is used;

- The simulations are started with the flow at rest.

CFX-4.3 is a finite-volume based code using structured non-staggered multi-block grids. Numerical grids were generated for the computational domain. For each simulation with specific $l / h, \alpha$ and $\beta$ values, the numerical grids are refined until a grid independent solution is obtained.

The local mass transfer coefficient follows from the well-known film model:

$k=\frac{1}{C_{\mathrm{b}}-C_{\mathrm{w}}}\left(-\mathcal{D} \frac{\partial C}{\partial y}\right)_{\text {wall }}$

The mass transfer coefficient averaged over the membrane area is:

$k_{\mathrm{m}}=\frac{1}{A} \int_{A} k \mathrm{~d} A$
It is clear from Eq. (16) that, in present study, $k_{\mathrm{m}}$ is only averaged over the membrane area. In 3D simulations, calculation of mass transfer coefficients averaged over both area and time with the CFX software is very time consuming and hardly worthwhile, since the fluctuations of the mass transfer coefficients averaged over area are $<2 \%$ at $R e=100$ and $<5 \%$ at $R e=500$.

\section{Results and discussion}

\subsection{Flow pattern and mechanism of enhanced mass transfer}

Due to the presence of spacers, the flow in the channel becomes unsteady at moderate Reynolds numbers. In the present research, it was found that vortex shedding happens at $\operatorname{Re}>150$; however, the critical Reynolds numbers for the start of vortex shedding are dependent on $l / h, \alpha$ and $\beta$. This phenomenon is in agreement with the result of flow visualization in flat channels equipped with round bars perpendicular to the mean flow direction [22].

Fig. 4 shows the velocity vectors in the plane $z / l=$ 0.5 , parallel to the mean flow direction in the spacer channel. It can be seen that the flow in channels filled with net spacers is characterized by the presence of transversal vortices with axes normal to the mean flow direction immediately in front and behind the filaments. Local maxima of Sherwood numbers exist in these areas. In Fig. 5, the local Sherwood numbers on the lower wall $(y=0)$ are shown. The local Sherwood numbers on the upper wall $(y=h)$ are presented in Fig. 6. It can be easily seen from Figs. 5 and 6 that local maxima of the Sherwood numbers exist in both the $x$ - and $z$-directions.

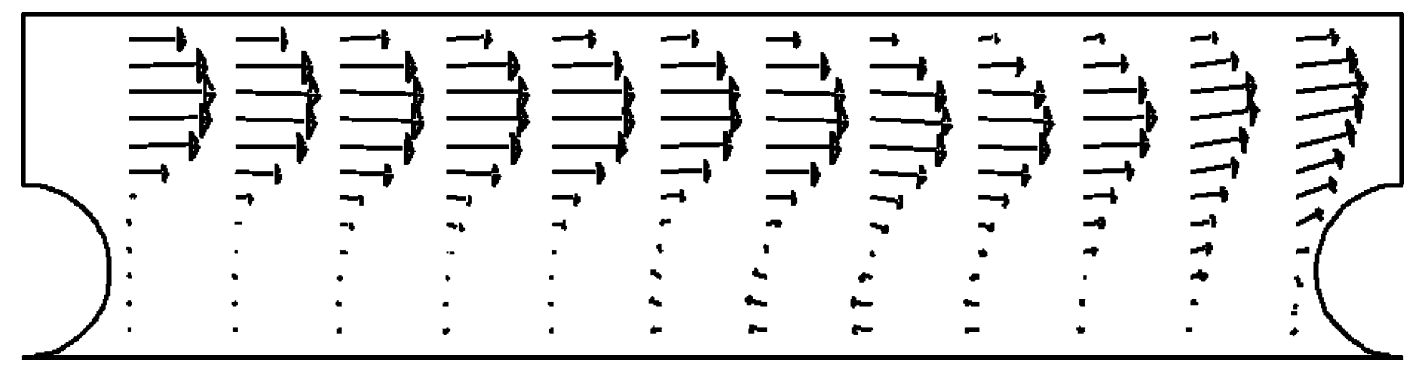

Fig. 4. Vector plot of flow at plane $z / l=0.5$ at $R e=188$ (spacer parameters: $l / h=4, \alpha=0^{\circ}$ and $\beta=90^{\circ}$ ). 


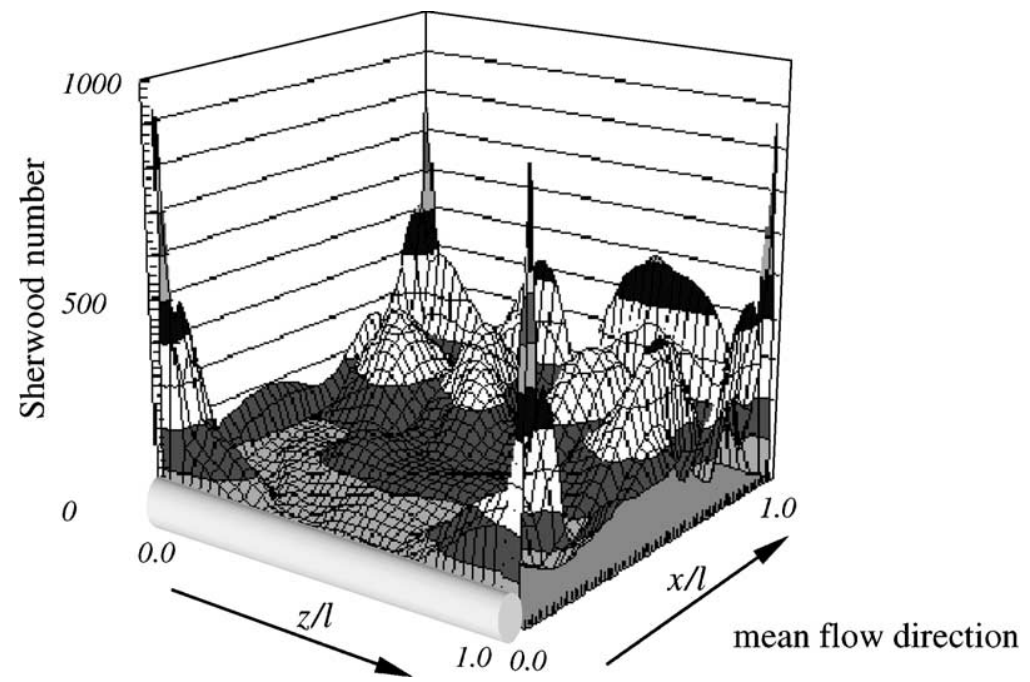

Fig. 5. Local Sherwood numbers on the lower wall at $R e=188$ (spacer parameters: $l / h=4, \alpha=0^{\circ}$ and $\beta=90^{\circ}$ ).

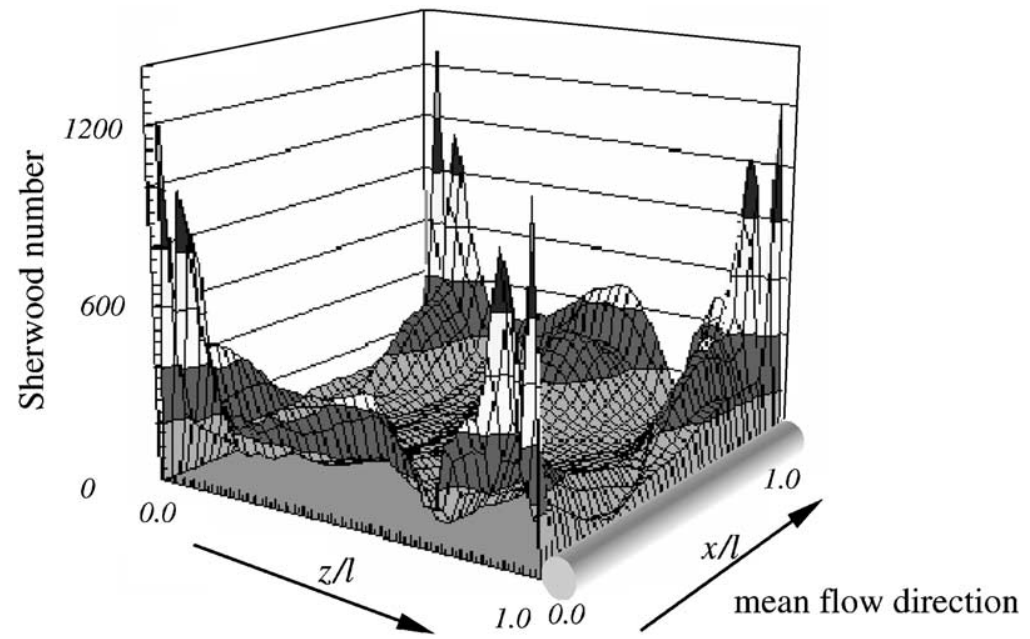

Fig. 6. Local Sherwood numbers on the upper wall $(y=h)$ at $R e=188$ (spacer parameters: $l / h=4, \alpha=0^{\circ}$ and $\beta=90^{\circ}$ ).

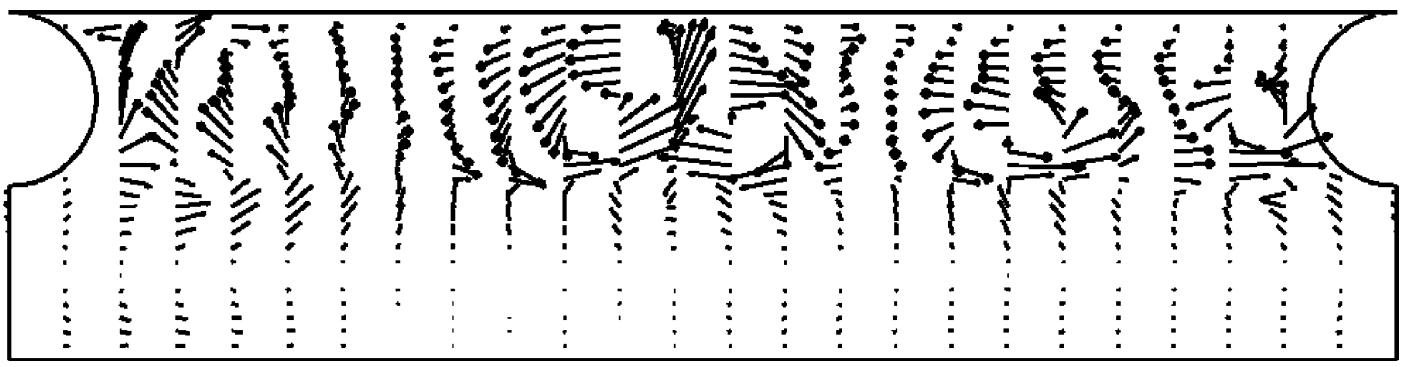

Fig. 7. Vector plot of flow at plane $x / l=0.0625$ at $R e=350$ (spacer parameters: $l / h=4, \alpha=0^{\circ}$ and $\beta=90^{\circ}$ ). 


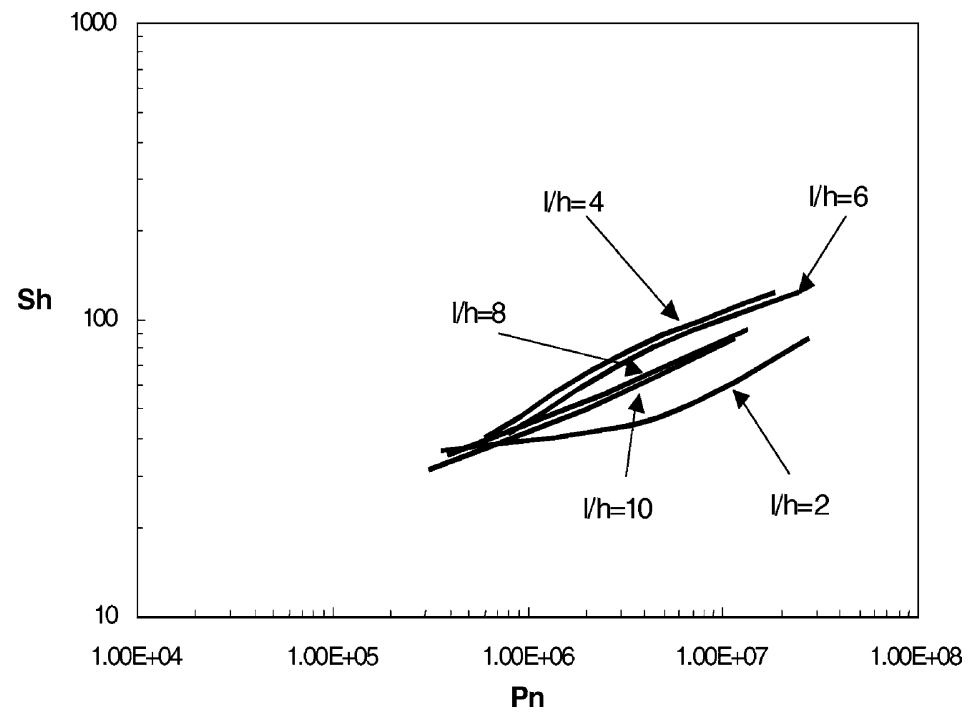

Fig. 8. Sh as function of $P n$ at different values of $l / h\left(\alpha=0^{\circ}\right.$ and $\left.\beta=90^{\circ}\right)$.

A comparison of the Sherwood numbers on the two walls indicates that the maxima on the upper wall are higher due to the following phenomena:high local velocities in areas with filaments perpendicular to the mean flow direction; andlongitudinal vortices (axis in the mean flow direction) as shown in the vector plot in a plane perpendicular to the mean flow direction in Fig. 7. This flow pattern is similar to the "cork-screw flow" observed by Zimmerer and Kottke [23] in spacer-filled channel with visualization experiments. In Fig. 7, $R e=350$, since this phenomenon is more pronounced at higher Reynolds numbers. It should be noted here that studies using 2D flow models in spacer-filled channels do not reveal the 3D longitudinal vortices as shown previously.

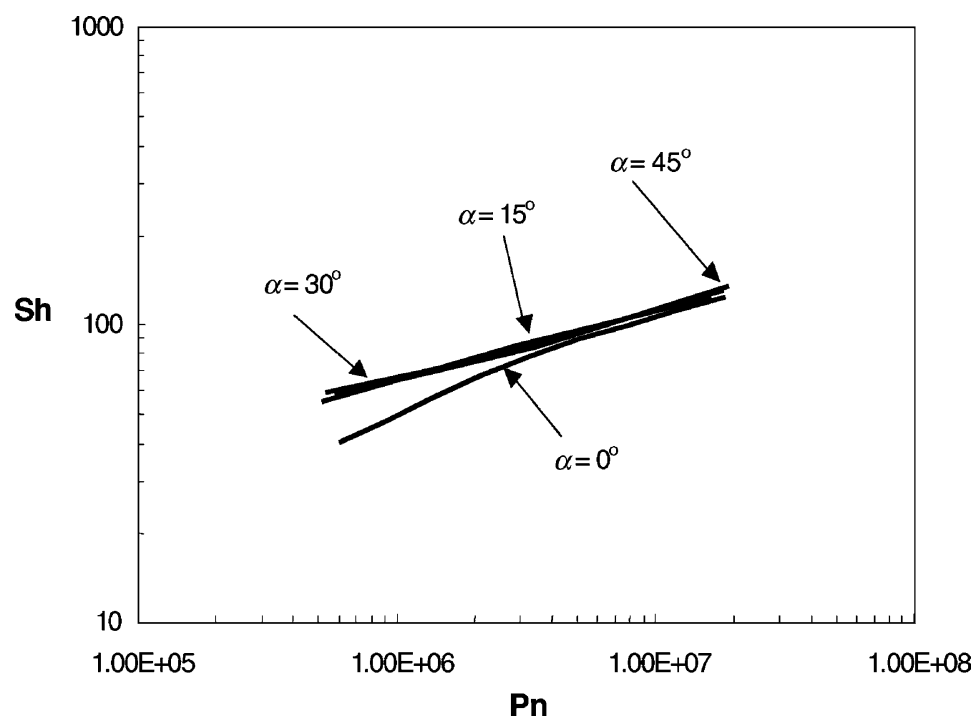

Fig. 9. Sh as function of $P n$ at different values of $\alpha\left(l / h=4\right.$ and $\left.\beta=90^{\circ}\right)$. 


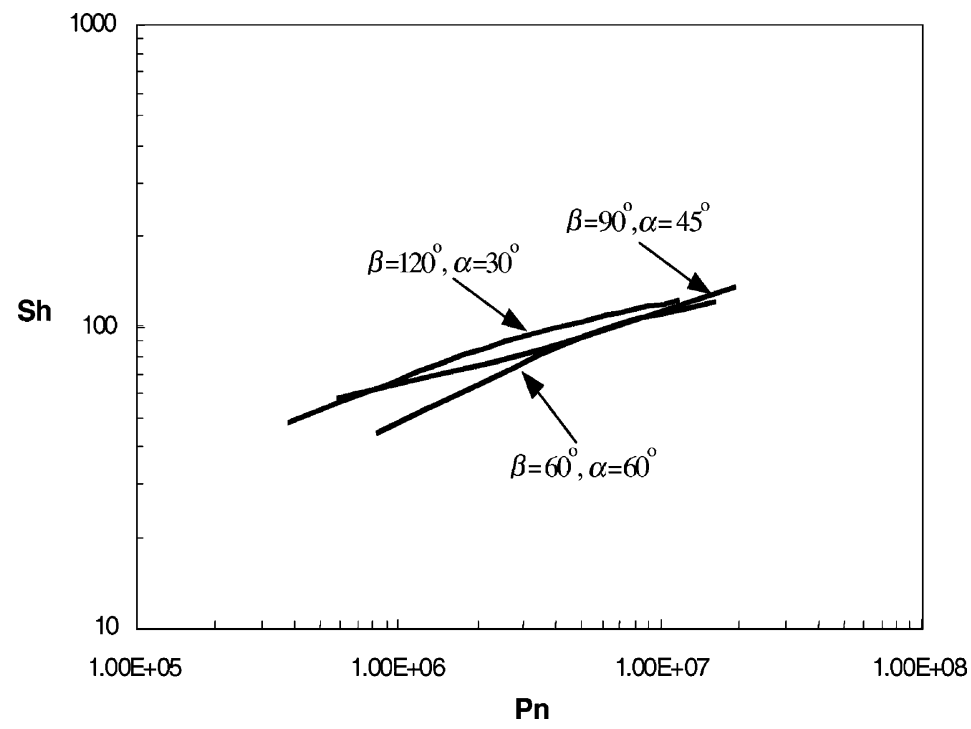

Fig. 10. $S h$ as function of $P n$ at different values of $\beta(l / h=4)$.

5.2. The influence of $l / h, \alpha$ and $\beta$ on spacer performance

Comparison of the performance of spacers with different geometric parameters was carried out on the basis of $S h$ versus $P$ n. Fig. 8 shows the Sherwood number, $S h$, as a function of the power number, $P n$, for $\alpha=0^{\circ}$ and $\beta=90^{\circ}$. The parameter $l / h$ appears to have a significant influence on mass transfer, with $l / h$ around 4 being the optimal value.

The influence of the flow attack angle $\alpha$ on spacer performance is shown in the $S h$ versus $P n$ plot of Fig. 9

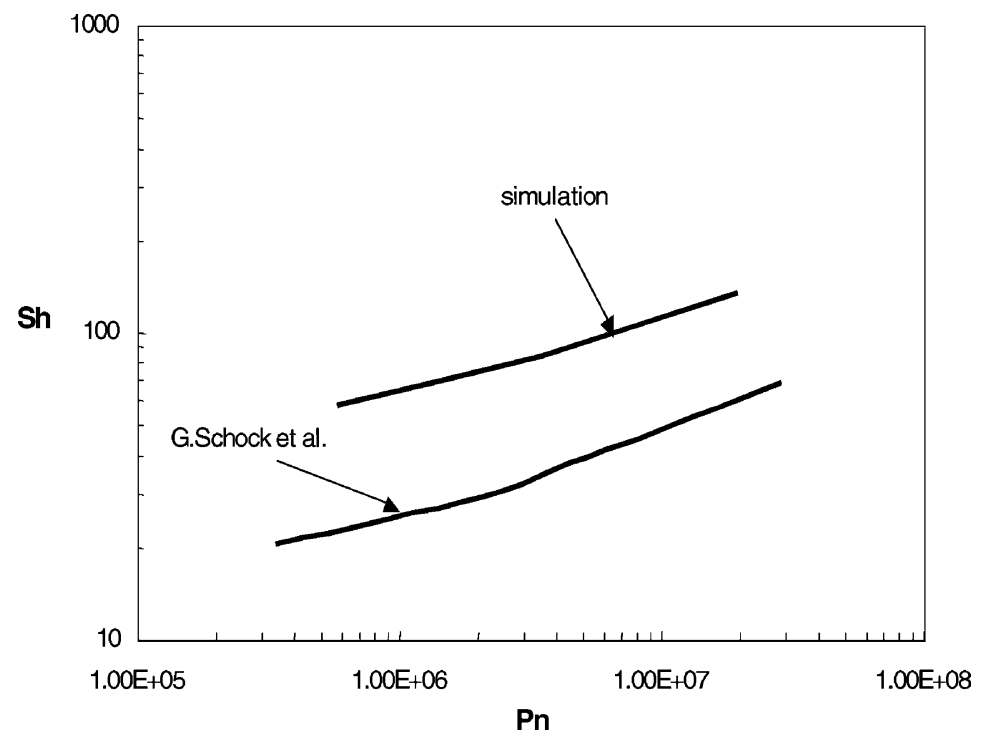

Fig. 11. Comparison between the results of the present CFD simulations and Schock and Miquel [12] $\left(l / h=4, \alpha=45^{\circ}\right.$ and $\left.\beta=90^{\circ}\right)$. 


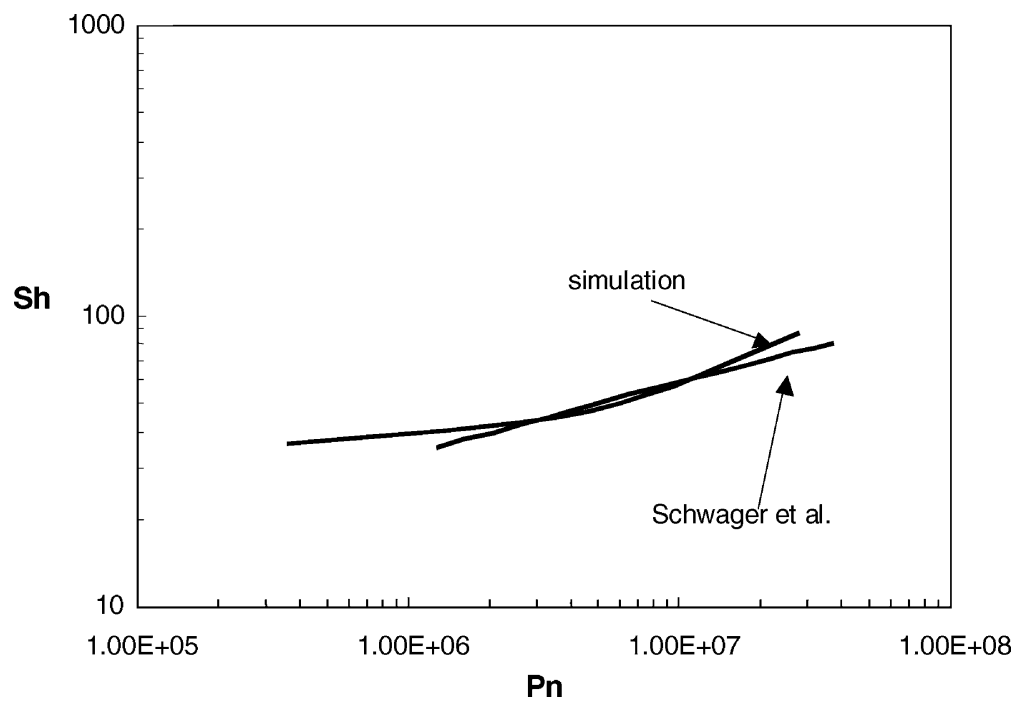

Fig. 12. Comparison between the present CFD simulations $\left(l / h=2, \alpha=0^{\circ}\right.$ and $\left.\beta=90^{\circ}\right)$ and the measurements of Schwager et al. $\left(l / h=1.3-1.8, \alpha=0^{\circ}\right.$ and $\left.\beta=90^{\circ}\right)[6]$.

for $l / h=4$ and $\beta=90^{\circ}$. Setting $\alpha=15,30$ and $45^{\circ}$ improves the spacer performance over the lower $P n$ range.

The influence of $\beta$ is as shown in Fig. 10. In order to limit the number of numerical simulations only three cases were considered: $\beta=60,90$ and $120^{\circ}$ while keeping $\beta+2 \alpha=180^{\circ}$. An improved performance is shown for $\beta=120^{\circ}$.
The above simulation results have been compared with results reported in the literature. Although a number of papers on net spacers have been published, only two reports on non-woven net spacers of the above geometries were found: the papers of Schock and Miquel [12] and Schwager et al. [6].

Schock and Miquel [12] have obtained their correlations from experiments with spacers of different $l / h$

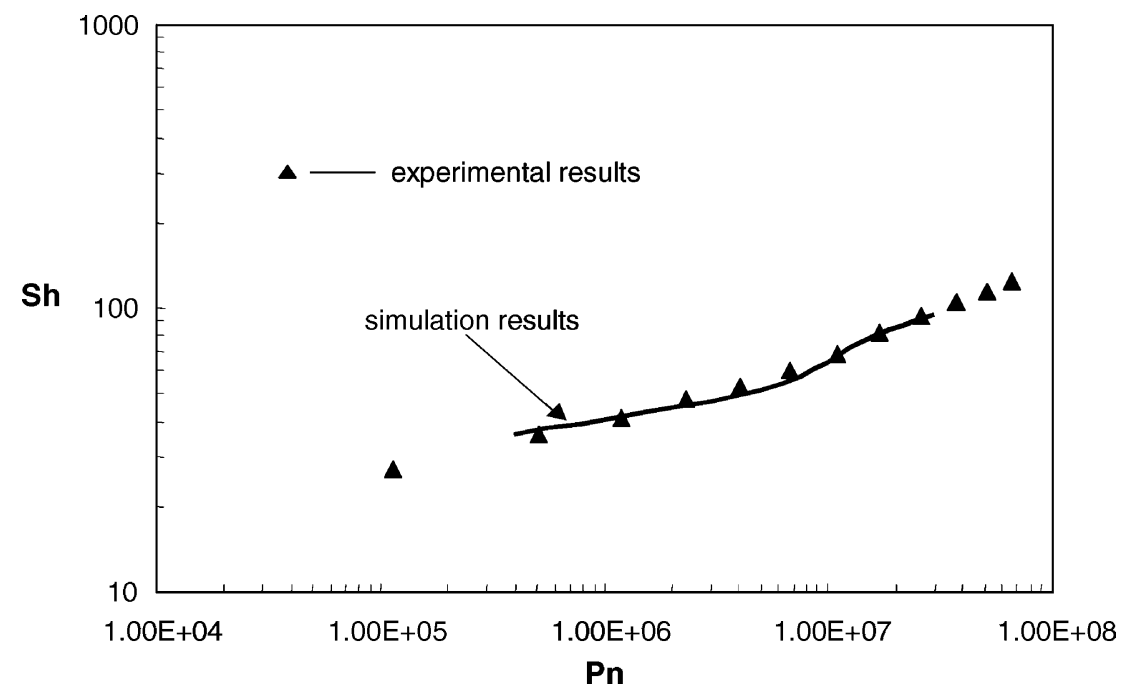

Fig. 13. Comparison of the present CFD simulation $\left(l / h=2.2, \alpha=0^{\circ}\right.$ and $\left.\beta=90^{\circ}\right)$ with experiments $\left(l / h=2.2, \alpha=0^{\circ}\right.$ and $\left.\beta=90^{\circ}\right)$. 


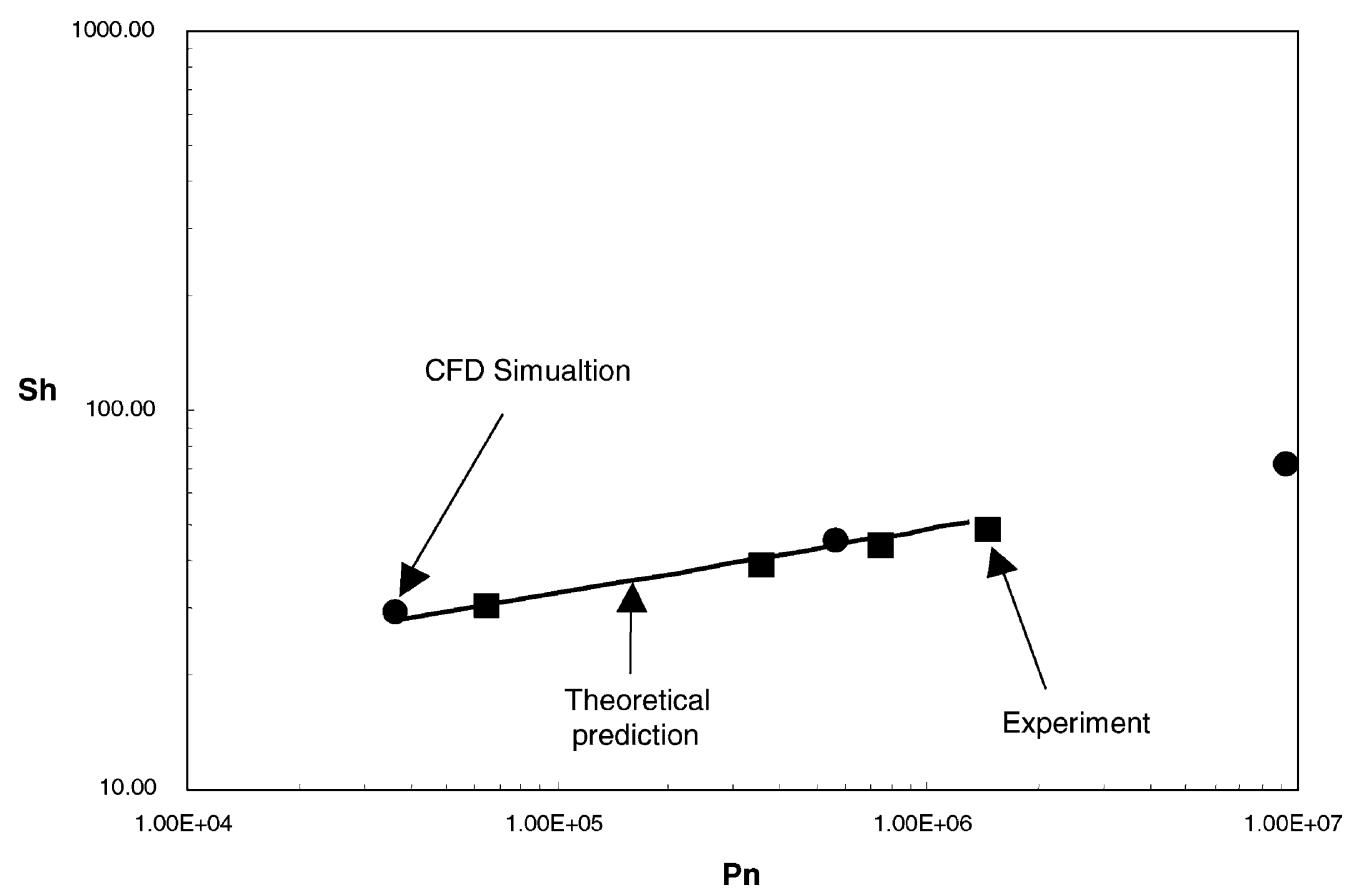

Fig. 14. Comparison among experiment, theoretical prediction and CFD simulation in an empty channel with $L / h=10$.

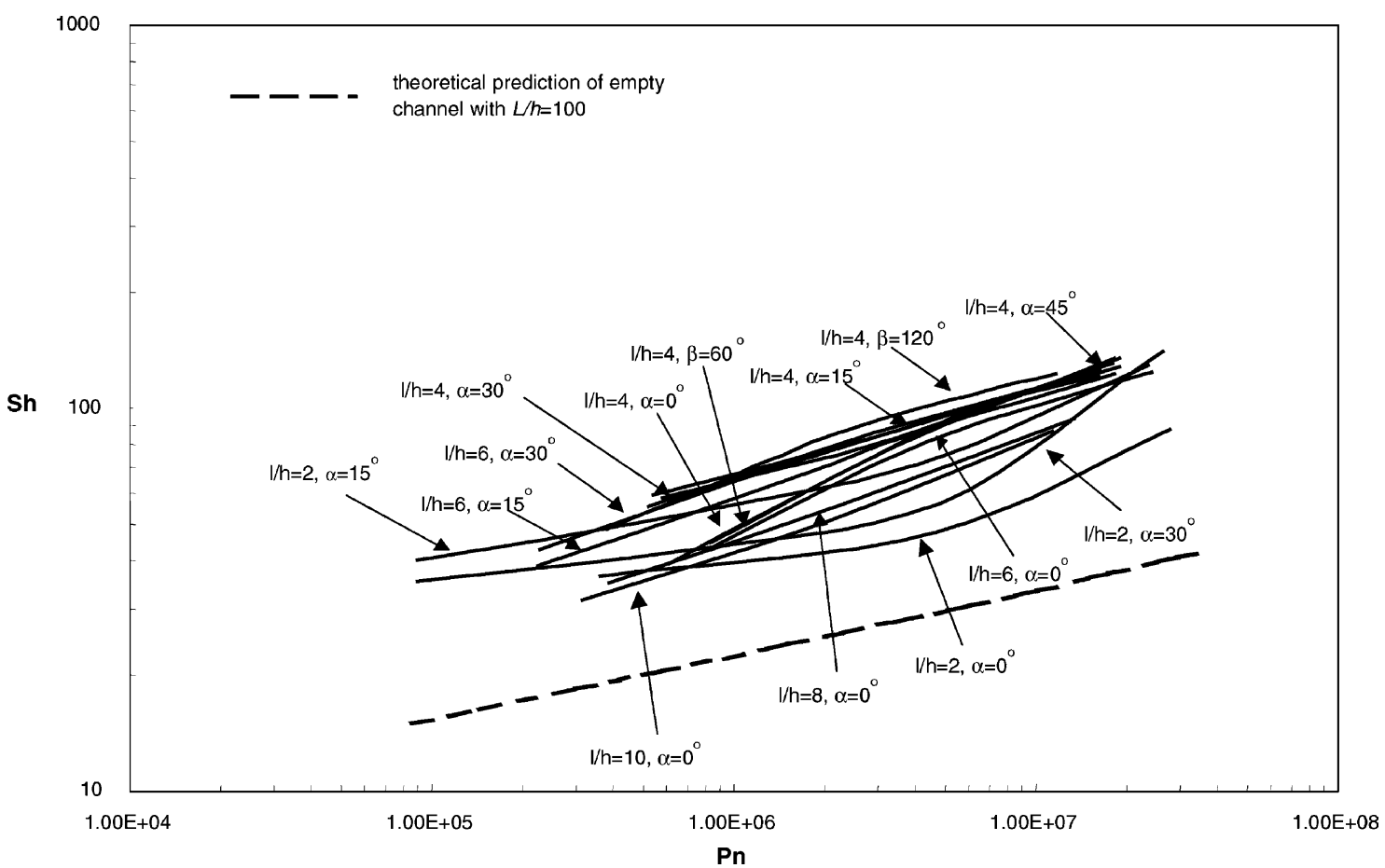

Fig. 15. Plot of all non-woven spacer simulation data at different values of $l / h, \alpha$ and $\beta$ and empty channel data. 
ranging from 3.6 to 4.3 using the osmotic pressure method. All spacers were non-woven with $\alpha=45^{\circ}$. Fig. 11 shows the wide gap between the results of the present CFD simulations at $l / h=4, \alpha=45^{\circ}$ and $\beta=$ $90^{\circ}$ and those of Schock and Miquel [12]. The explanation may be that the calculation of mass transfer coefficients by the osmotic pressure method has been made by using a simplified filtration model. In a study of van der Waal and Rácz [4], it was also found that the results calculated from similar osmotic pressure measurements in an empty flat channel were considerably lower than the theoretical predictions. The empirical correlations from Schwager et al. [6] follow from their mass transfer measurements using the electrochemical (limiting current) method. The results of Schwager for values of $l / h$ ranging from 1.3 to 1.8 , $\alpha=0^{\circ}$ and $\beta=90^{\circ}$ are compared with the present simulations at $l / h=2, \alpha=0^{\circ}$ and $\beta=90^{\circ}$. The lines for the two cases, as shown in Fig. 12, are in close agreement.

In order to validate the results of the present CFD simulations, mass transfer rates were measured using the electrochemical method. Complete and detailed results of these experiments will be presented in a future paper. However, a comparison of some of these experimental results with those from CFD simulations is as shown in Fig. 13. It is clear from Fig. 13 that the results from CFD simulation and experiment are in good agreement.

Measurements of Sherwood numbers in empty channels with $L / h=10$ are compared with CFD calculations and theory in Fig. 14. The well-known Graetz equation predicts the Sherwood numbers for empty channel flow in the laminar region:

$S h_{\mathrm{empty}}=1.47\left(\operatorname{ReSc} \frac{h}{L}\right)^{1 / 4}$ for $\operatorname{ReSc} \frac{h}{L}>20$

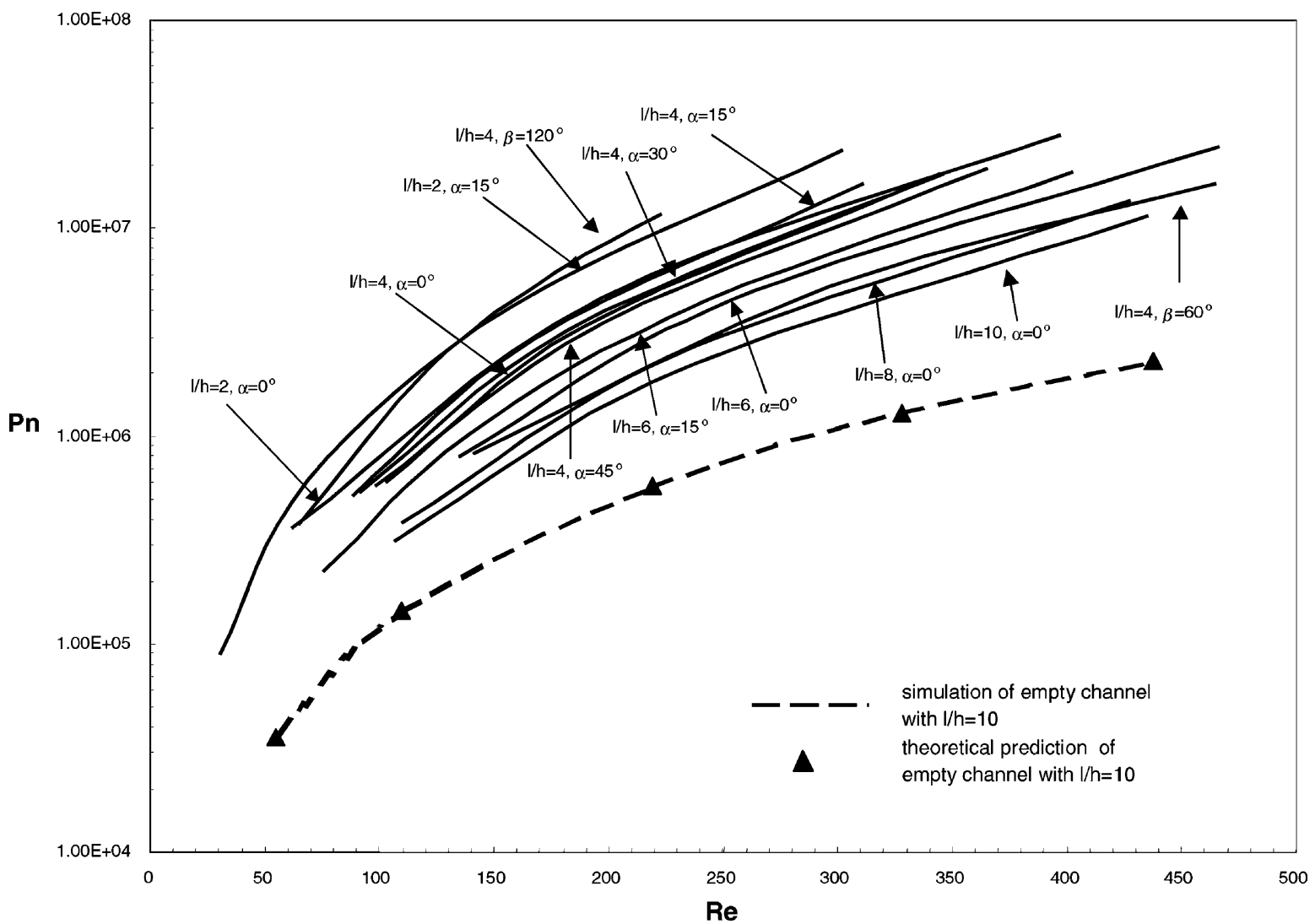

Fig. 16. Plot of all non-woven spacer power number data and empty channel data as a function of $R e$. 
and the power number, $P n$, follows from:

$P n_{\mathrm{empty}}=E u \frac{h}{L} R e^{3}=12 R e^{2}$

Fig. 14 shows that the agreement is excellent.

\subsection{Optimization of spacers}

A comprehensive survey of all present CFD simulations for spacers with different values of $l / h, \alpha$ and $\beta$ and for empty channels is as shown in Fig. 15. If there is no specific indication of $\beta$, the spacers presented in Figs. 15 and 16 have $\beta=90^{\circ}$.

The results indicate that there is no geometry that is optimal for all values of $P n$. For instance, the performance of the spacers with $l / h=4, a=30^{\circ}$ and $\beta=90^{\circ}$ is slightly superior to the spacers with $l / h=$ 4, $a=30^{\circ}$ and $\beta=120^{\circ}$ at low Pn.

Fig. 16 gives a survey of all simulated spacer power data. The power number at a certain value of the geometric parameters $l / h, \alpha$ and $\beta$ is an unique function of the Reynolds number, $R e$. The power number increases with increasing $R e$. As expected, the spacers in the channel cause an extra pressure drop (power consumption) over the channel.

In spiral wound module, the ratio of $L / h$ can be up to 1000 . From Fig. 15, it is obvious that an empty flat channel with $L / h=100$ is already less effective than a spacer-filled channel. This finding confirms again the essential role spacers have in industrial spiral wound modules.

\section{Conclusions}

Sherwood numbers, $S h$, and power numbers, $P_{n}$, were calculated using CFD simulations with 3D transient models for low-Reynolds flows in flat channels filled with non-woven spacers. The simulations show transversal and longitudinal vortices, vortex shedding and instable flow behavior, which is in agreement with earlier studies of flow visualization in spacer-filled channels [22,23].

Fig. 15 shows clearly mass transfer optima as a function at certain values of $l / h$. Results from CFD simulations indicate that if $l / h$ is small (i.e. $l / h=2$ ), the flow between two neighboring filaments consists mainly of one "dead eddy", which does not contribute much to mass transfer. At moderate value of $l / h$, i.e. $l / h=4$, the flow between neighboring filaments is characterized by shedding of vortices which will effectively enhance mass transfer. If the value of $l / h$ is very high, i.e. $l / h=10$, shedding of vortices will only enhance mass transfer in the direct neighborhood of the filaments, resulting in lower average mass transfer. This means that an optimum value of $l / h$ exists. The geometric parameters, $\alpha$ and $\beta$, also play important roles in enhancing mass transfer. In the present study, it was found that the spacer geometry is optimal for $l / h=4, a=30^{\circ}$ and $\beta=120^{\circ}$ in a large range of Pnvalues.

Good agreement was noted between the results of the CFD simulations and the experimental data reported in the literature. Also good agreement was found between the simulated empty channel data and theory.

The results of the CFD simulations were validated using a few experimental data obtained by the authors of the present paper. A future paper will describe these experiments in more detail.

\section{Acknowledgements}

The support of STW, the Dutch Technology Foundation, is gratefully acknowledged.

\section{References}

[1] B.B. Gupta, J.A. Howell, D. Wu, R.W. Field, A helical baffle for cross-flow microfiltration, J. Membr. Sci. 99 (1995) $31-42$.

[2] H.M. Yeh, K.T. Chen, Improvement of ultrafiltration performance in tubular membranes using a twisted wire-rod assembly, J. Membr. Sci. 178 (2000) 43-53.

[3] H.R. Millward, B.J. Bellhouse, I.J. Sobey, The vortex membrane bio-reactor: hydrodynamics and mass transfer.

[4] M.J. van der Waal, I.G. Racz, Mass transfer in corrugatedplate membrane modules. Part I. Hyperfiltration experiments, J. Membr. Sci. 40 (1989) 243-260.

[5] D.S. Fischl, K.J. Hanson, R.H. Muller, C.W. Tobias, Mass transfer enhancement by small flow obstacles in electrochemical cells, Chem. Eng. Commun. 38 (1984) 191-207.

[6] F. Schwager, P.M. Robertson, N. Ibl, The use of eddy promoters for the enhancement of mass transport in electrolytic cells, Electrochim. Acta 25 (1980) 1655-1665.

[7] F.B. Leitz, L. Marinčić, Enhanced mass transfer in electrochemical cells using turbulence promoters, J. Appl. Electrochem. 7 (1977) 473-484. 
[8] M.S. Isaacson, A.A. Sonin, Sherwood number and friction factor correlations for electrodialysis systems, with application to process optimization, Ind. Eng. Chem., Process Des. Dev. 15 (1976) 313-321.

[9] C.H. Wang, Technology review-a survey of recent patents of fin-and-tube heat exchangers, J. Enhanced Heat Transfer 7 (2000) 333-345.

[10] M. Fiebig, Vortex generators for compact heat exchangers, J. Enhanced Heat Transfer 2 (1995) 43-61.

[11] J. Fárková, The pressure drop in membrane module with spacers, J. Membr. Sci. 64 (1991) 103-111.

[12] G. Schock, A. Miquel, Mass transfer and pressure loss in spiral wound modules, Desalination 64 (1987) 339352.

[13] J.J.S. Shen, R.F. Probstein, Turbulence promotion and hydrodynamic optimization in an ultrafiltration process, Ind. Eng. Chem., Process Des. Dev. 18 (1979) 547-554.

[14] Y. Winograd, A. Solan, M. Toren, Mass transfer in narrow channels in the presence of turbulence promoters, Desalination 13 (1973) 171-186.

[15] W.G. Light, T.V. Tran, Improvement of thin-channel design for pressure-driven membrane systems, Ind. Eng. Chem., Process Des. Dev. 20 (1981) 33-40.

[16] Z. Cao, D.E. Wiley, A.G. Fane, CFD simulation of net-type turbulence promoters in a narrow channel, J. Membr. Sci. 185 (2001) 157-176.
[17] S.K. Karode, A. Kumar, Flow visualization through spacer filled channels by computational fluid dynamics. Part I. Pressure drop and shear rate calculations for flat sheet geometry, J. Membr. Sci. 193 (2001) 69-84.

[18] A.R. Da Costa, A.G. Fane, C.J.D. Fell, A.C.M. Franken, Optimal channel spacer design for ultrafiltration, J. Membr. Sci. 62 (1991) 275-291.

[19] A. Storck, D. Hutin, Energetic aspects of turbulence promotion applied to electrolysis processes, Can. J. Chem. Eng. 58 (1980) 92-102.

[20] M. Zlokarnik, Dimensional Analysis and Scale-up in Chemical Engineering, Springer, Berlin, 1991.

[21] M.S. Issacson, A.A. Sonin, Sherwood number and friction factor correlations for electrodialysis systems, with application to process optimization, Ind. Eng. Chem., Process Des. Dev. 15 (1976) 313-321.

[22] P. Feron, Hydrodynamics and mass transfer in obstructed narrow channels, Ph.D. thesis, Cranfield Institute of Technology, 1991.

[23] C.C. Zimmerer, V. Kottke, Effects of spacer geometry on pressure drop, mass transfer, mixing behavior and residence time distribution, Desalination 104 (1996) 129-134.

[24] Z.X. Yuan, W.Q. Tao, Q.W. Wang, Numerical prediction for laminar forced convection heat transfer in parallel plate channels with streamwise-periodic rod disturbances, Int. J. Numerical Meth. Fluids 28 (1998) 1371-1387. 